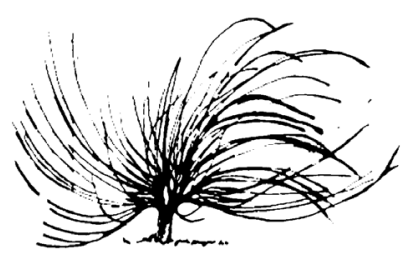

\title{
M-learning y desarrollo de habilidades digitales en educación superior a distancia
}

\author{
Mariana Figueroa de la Fuente ${ }^{l}$ \\ Universidad de Quintana Roo \\ México \\ mfigueroa@uqroo.edu.mx \\ Leonardo David Glasserman Morales ${ }^{2}$ \\ Tecnológico de Monterrey \\ México \\ glasserman@itesm.mx \\ María Soledad Ramírez Montoya ${ }^{3}$ \\ Tecnológico de Monterrey \\ México \\ solramirez@itesm.mx
}

\begin{abstract}
Resumen
El aprendizaje mediado por la tecnología ha requerido el desarrollo de nuevas habilidades para enseñar y construir el conocimiento. Aunada a esto, la introducción de los dispositivos móviles como herramienta de enseñanza ha aportado flexibilidad y libertad sin precedentes, revolucionado las prácticas educativas del siglo XXI. En este contexto, el presente estudio cualitativo muestra
\end{abstract}

\section{(c) (i) $(9)$}

Recibido: 1 de febrero de 2018. Aprobado: 24 de setiembre de 2018.

http://dx.doi.org/10.15359/rep.13-2.5

1 Profesora investigadora de la Universidad Quintana Roo en Playa del Carmen, México. Maestra en Tecnología Educativa del Tecnológico de Monterrey. Doctorante en Liderazgo y dirección de instituciones de educación superior por la Universidad Anáhuac del Norte.

2 Profesor investigador en la Escuela de Humanidades y Educación del Tecnológico de Monterrey.

3 Profesora investigadora titular y decana asociada de posgrado en la Escuela de Humanidades y Educación del Tecnológico de Monterrey. 
los resultados de implementar estrategias de m-learning en un ambiente virtual de aprendizaje, con el objetivo de analizar el proceso de desarrollo de habilidades digitales. De igual forma, busca determinar si es posible incrementar la motivación de los estudiantes, mediante la integración de actividades interactivas de aprendizaje (storylines) con la herramienta de tecnología educativa llamada Sharable Content Object Reference Model (SCORM). La pregunta que guió la investigación fue: ¿Cómo desarrolla las estrategias de aprendizaje del m-learning la alfabetización digital, en un curso de educación a distancia? Entre los principales hallazgos se encontró: a) la alfabetización digital se desarrolla al incluir recursos didácticos vinculados a la tecnología móvil; b) el uso de los dispositivos móviles apoya en estrategias didácticas que promueven el desarrollo de habilidades digitales; y c) la tecnología SCORM puede incrementar la motivación de los estudiantes, al apoyar el proceso educativo virtual de forma interactiva.

Palabras claves: alfabetización digital, ambientes virtuales de aprendizaje, habilidades digitales, aprendizaje móvil, tecnología educativa.

\begin{abstract}
Learning with technology has required the development of new abilities to teach and build knowledge. In addition, the introduction of mobile devices as a teaching tool has provided an unprecedented freedom and flexibility, which has revolutionized the educational practices of the $21 \mathrm{st}$ century. In this context, this qualitative study shows the results of implementing m-learning strategies in a virtual learning environment, with the aim of analyzing the process of development of digital abilities to implement strategies linked to the use of mobile technology in a distance course. In addition, it sought to determine if it is possible to increase the student's motivation by integrating interactive learning activities called storylines with the Sharable Content Object Reference Model (SCORM) educational tool. Therefore, the question that guided the investigation was: How do $\mathrm{m}$-learning strategies develop digital literacy in a distance education course? The results show that: a) digital literacy
\end{abstract}


is developed by including teaching resources related to mobile technology, based on educational theories; b) the use of mobile devices supports teaching strategies that promote the development of digital skills; c) the SCORM technology can enhance students motivation, to support the virtual educational process graphically and interactive.

Keywords: digital literacy, virtual learning environments, digital skills, m-learning; educational technology.

\section{Introducción}

$\mathrm{L}$ as tecnologías de la información y la comunicación (TIC), las tendencias de la web 2.0 y el uso generalizado de tecnologías como dispositivos móviles han revolucionado en las últimas dos décadas el comercio tradicional, las industrias soportadas por bases de datos como la música, diferentes tipos de software y periódicos, así como el sector educativo que se ha centrado en la información, por lo que inevitablemente tiende a la digitalización. La nueva generación educativa a distancia o e-learning, basada en modelos de negocio, ha surgido y ha comenzado a ser tan eficaz y eficiente como comercio electrónico o e-commerce (Pathak, 2016), por lo que se ha vuelto necesario proporcionar nuevas soluciones para satisfacer las necesidades de los alumnos, las cuales permitan situarlos en el centro del proceso de aprendizaje y darles libertad de elegir el tipo de dispositivos que usarán con especial atención a las tecnologías móviles (Conde, García-Peñalvo, Alier y Piguillem, 2013).

En este sentido, la Organización de las Naciones Unidas para la Educación, la Ciencia y la Cultura (UNESCO) recomienda que la educación superior centre sus esfuerzos en la internacionalización, el incremento de la oferta de programas en línea, el perfeccionamiento del diseño de educación a distancia y el uso de las TIC con fines académicos, principalmente en los países en desarrollo, para afrontar los cambios de la sociedad del conocimiento de forma competitiva (Didou, 2014).

Por lo dicho, la educación requiere adaptarse a nuevas modalidades que permitan, por un lado, gestionar el conocimiento dentro de las empresas e instituciones, para desarrollar nuevas soluciones que mejoren los sistemas de información (García-Holgado y García-Peñalvo, 2016); por el otro, hacer uso efectivo y ético de las tecnologías emergentes y aprovechar las ventajas de las TIC, el desarrollo de los dispositivos 
móviles, el aumento de la conectividad, el impacto de las redes sociales, entre otros factores que han abierto el debate académico sobre su uso en la tecnología educativa y remarcan la importancia de los entornos personales de aprendizaje (PLE) (Barroso, Cabero y Vázquez, 2012).

Para lo anterior, resulta necesario vencer los desafíos iniciales del uso, perfeccionamiento, gestión y promoción de las tecnologías, pues siempre generan incertidumbre en el usuario. Sin embargo, se ha demostrado que, si se gestionan correctamente y desarrollan su potencial, los resultados pueden ser sustanciales, ya que permiten agregar valor a los productos o servicios (Jiménez-Hernández, Castellanos-Domínguez y Villa-Enciso, 2011). Como se ha comprobado en diversos estudios, entre los que destaca el caso del eLearning knowledge quality (KQ) (en el que Waheed (2016) demostró la relación positiva existente entre la educación de calidad recibida por alumnos de un curso en línea y su percepción de satisfacción del curso, el aumento de su rendimiento académico y, en consecuencia, el incremento de su lealtad al aprendizaje en línea), se puede afirmar que la calidad del conocimiento contribuye al éxito de su sistema de gestión.

De ahí que sea relevante fomentar la aplicación de las tecnologías en la educación y perfeccionar el uso de las herramientas didácticas utilizadas en ambientes digitales, siempre fundamentadas en teorías y estrategias educativas para el diseño de los recursos m-learning (Ramos, Herrera y Ramírez, 2010), con un enfoque centrado en el alumno y en el desarrollo tanto de competencias como de capacidades digitales, en el que el aprendizaje, el autodesarrollo y el bienestar deben desempeñar un papel fundamental (Zezulkova, 2017). Lo antes dicho se debe a que, en esencia, los objetivos, el uso y la integración que se dé a las tecnologías es lo que permite maximizar su potencial, diversificar sus usos y crear ambientes de comunicación novedosos, no la tecnología aislada por sí misma. En este sentido, el m-learning es una metodología de enseñanza-aprendizaje que en los últimos años ha logrado demostrar ser una aliada del sector educativo para vencer algunas problemáticas, al permitir el acceso al conocimiento en el momento adecuado, interactivo, cooperativo, portátil y personalizado, con lo que facilita el aprendizaje formal e informal y, por tanto, podría transformar la experiencia tanto de la educación, como de la capacitación profesional y, eventualmente, alcanzar la educación para todos o EPT (UNESCO, 2016). 
Además, por ser una poderosa herramienta en la educación, al impulsar el aprendizaje y la enseñanza de forma novedosa, el m-learning puede ayudar a cerrar la brecha digital en la sociedad del conocimiento (UNESCO, 2011), pues ofrece flexibilidad en el proceso de aprendizaje y rompe las barreras del tiempo y del espacio, lo que constituye características únicas que despiertan en los educandos la motivación por aprender (Ciampa, 2013). De igual forma, surgen factores que ofrecen ventajas competitivas para el rediseño del ambiente de enseñanza-aprendizaje y revolucionar los sistemas instruccionales.

Asimismo, el sistema educativo necesita proveer las herramientas necesarias a las nuevas generaciones, para que puedan insertarse en el mercado laboral y enfrentar una competencia global, en donde las competencias digitales e informacionales son altamente valoradas, como la capacidad de buscar, filtrar, comprender, reproducir y compartir información digital. Solo así los países en vías de desarrollo estarán en condiciones de seguir los pasos de los más avanzados y ser partícipes de los adelantos de la tecnología, al igual que poder participar en los beneficios de la apertura del conocimiento, mediante la utilización de recursos educativos abiertos (REA) que abonan a reducir la brecha digital, como los repositorios abiertos, el uso de open courseware y los cursos masivos abiertos en línea (Massive Open Online Courses, por sus siglas en inglés). Sin embargo, las investigaciones de aprendizaje móvil en materia de educación se encuentran aún en fases tempranas de desarrollo, hace falta ahondar en las habilidades digitales que se desenvuelven, en cómo enseñarlas y evaluarlas, además de conocer cómo las tecnologías están modificando la calidad de la educación a distancia en ambientes virtuales. De aquí la importancia de los resultados de la presente investigación, para sumar a la comprensión de este fenómeno educativo.

Por tanto, en este estudio se planteó el objetivo de contribuir a la comprensión de la forma en que se desarrolla la alfabetización digital, por medio de estrategias de aprendizaje que emplean tecnología móvil en un curso de educación en línea. Para ello, se analizó el proceso de desarrollo de habilidades digitales en un ambiente virtual de aprendizaje, en el que se emplearon estrategias didácticas de tecnología móvil. 


\section{Antecedentes}

Casos de estudio que han documentado dichas innovaciones son, por ejemplo, los de Zatarain y Barrón (2011), quienes exploraron una propuesta metodológica, cuyo objetivo principal fue la identificación de estilos de aprendizaje utilizando un método de mapas autoorganizados implementados para trabajar en dispositivos móviles. Entre los hallazgos, destacan que, en un ambiente de aprendizaje electrónico como EDUCA, se pueden obtener datos valorativos con la ayuda de la red neuronal y las evaluaciones que el tutor inteligente va efectuando al estudiante, lo cual permite la identificación del estilo de aprendizaje al cual va dirigido el material didáctico que el sujeto se encuentra estudiando y la evaluación que el estudiante tiene con tal material.

Asimismo, Álvarez y Álvarez (2012) realizaron un análisis semiótico comparativo entre ambientes virtuales de aprendizaje no intervenidos y entornos de aprendizaje en red en el marco de cursos intervenidos. Una de las observaciones principales de este tipo de análisis es que los aspectos organizativos de los cursos tienen que ver con la manera en que se construyen las disposiciones de entrada para un proceso de enseñanza y aprendizaje.

Otros estudios fueron realizados por Organista-Sandoval, Serrano-Santoyo, McAnally-Sala, y Lavigne (2013), quienes analizaron apropiación y usos educativos del teléfono celular; entre los resultados, destaca que los estudiantes tienen más facilidad para manejar los dispositivos, así como una mayor media de uso del teléfono celular que los docentes, sin embargo, la posesión de teléfono celular fue de $97 \%$ en ambos grupos. Igualmente, se encontró que los usos educativos del celular son limitados; los principales fueron para comunicación, manejo de información y organización. Concluyeron señalando que, si bien el teléfono celular cuenta con gran potencial pedagógico, es necesario incrementar la contribución en el conocimiento científico al respecto, para su adecuada incorporación al proceso educativo universitario.

En fechas más recientes, los investigadores Figueredo y Jiménez (2015) presentan, a partir de una revisión teórica de los principales modelos para el diseño de estrategias de aprendizaje móvil mediadas por TIC, un modelo propio con todos los elementos teórico-prácticos, a manera de guía, para que otros docentes e investigadores sean capaces de innovar, al llevar a la práctica dichas estrategias e implementarlas 
en congruencia con los elementos de su práctica pedagógica. El proyecto se llevó a cabo en la Universidad de la Sabana, en el marco de la maestría en informática educativa, y tuvo como objetivo el diseño de estrategias de aprendizaje móvil, para desarrollar competencias en el manejo de la información. Entre los principales hallazgos, los autores señalan tres principales formas para incorporar estrategias de aprendizaje móvil al proceso de enseñanza-aprendizaje: 1) como un apoyo al proceso del docente, al constituir un nuevo canal de comunicación; 2) como un complemento al proceso del docente, con actividades por realizar por los estudiantes fuera del espacio del aula, para que sean parte su formación; 3) para que el docente pueda incorporar una nueva e innovadora estrategia de aprendizaje.

Asimismo, se encuentra la disertación doctoral sobre entornos personales de aprendizaje móvil ( $m P L E$, por sus siglas en inglés), en la educación superior, elaborada por Humanante-Ramos, García-Peñalvo y Conde-González (2016), del Programa Doctoral de Formación en la Sociedad del Conocimiento de la Universidad de Salamanca en España. La investigación tuvo como objetivo estudiar los $m P L E$ en la Universidad Nacional de Chimborazo en Ecuador, como una propuesta por ser adoptada en educación superior, tomando en consideración el ya masivo uso que el estudiantado universitario hace en la actualidad de los dispositivos móviles. La metodología se basó en una revisión exhaustiva de trabajos teóricos previos, para conceptualizar los $m P L E$, y proponer, a partir de ella, una estructura diseñada por ser implementada en la realidad y así situar a los estudiantes en el centro de su proceso de aprendizaje, al darles la libertad de elegir los recursos y herramientas disponibles en la web, de acuerdo con sus necesidades, estilos de aprendizaje y preferencias. Los resultados de esta experiencia son favorables, ya que comprobaron dotar al alumnado de mayor personalización y accesibilidad de contenidos de aprendizaje, pero también se encontraron algunas limitantes como la accesibilidad de los móviles al Internet fuera de los campus universitarios y los costos de aquellos, que aún son elevados para los jóvenes universitarios.

Finalmente, González-Bañales y Monárrez-Armendáriz (2014), investigadores del Departamento de Sistemas y Computación, del Instituto Tecnológico de Durango, México, realizaron una indagación exploratoria de alcance y transversal, con el objetivo de investigar la incorporación de plantillas de diseño responsivo y el uso de redes 
sociales para acceder a los contenidos de la plataforma Moodle. Entre los principales hallazgos, se encontró que esta plataforma tiene la capacidad suficiente para responder correctamente y alojar componentes externos como Facebook, así como plantillas con diseño adaptativo y puede usarse independientemente de los navegadores, sistemas operativos y dispositivos móviles desde los cuales se acceda.

Recapitulando, como se pudo observar en los ejemplos anteriores, las estrategias didácticas vinculadas al m-learning son, actualmente, una realidad educativa que ha despertado el interés de investigadores y docentes, pero aún se requiere generar más información de casos positivos que ayuden a comprobar sus beneficios pedagógicos, para de esta forma impulsar la adopción de las nuevas tecnologías en todo el sistema educativo. A continuación, se muestran los referentes teóricos utilizados para definir los conceptos básicos que sirvieron de guía en este estudio: la alfabetización digital en educación a distancia y la innovación con aprendizaje móvil.

\section{Referentes teóricos}

La alfabetización digital en educación a distancia refiere al grado de dominio sobre las herramientas y recursos digitales para construir el conocimiento, por medio de una matriz tridimensional de atributos, habilidades y actitudes que dependen de ocho aspectos esenciales: cultural, cognitivo, edificante, comunicativo, confianza, creativo, crítico y cívico (Belshaw, 2012). Estos parámetros resultan indispensables para jerarquizar y dar sentido a los resultados de las consultas a los extensos volúmenes de información de la red y ser capaces de evaluar, integrar y reproducir información en diversos formatos digitales.

Dichas habilidades se pueden agrupar, por tipo de alfabetización, en cuatro categorías centradas en las siguientes áreas clave de trabajo académico: el aprendizaje y la enseñanza, las investigaciones, la comunicación y colaboración, así como la administración (Newland y Handley, 2016). Por tanto, en el campo educativo, se deberá garantizar el desarrollo de competencias para hacer uso de la información, por medio de recursos móviles en las estrategias de aprendizaje; además, incentivar a los docentes al diseño de recursos didácticos bajo tres premisas para que el aprendizaje sea permanente: flexibilidad en el tiempo, espacio y lugar (Cobo y Moravec, 2011). 
En este sentido, un aspecto importante es la evaluación, que en este caso requiere medir tres áreas del proceso de inclusión digital: las competencias informacionales, digitales y sociales. Para lograrlo, se debe diseñar modelo evaluativo capaz de medirlas, sin descuidar la valoración de otros importantes factores como el manejo de redes digitales, la lectura digital y la ética en el uso de las TIC (Cuevas y García, 2010). Igualmente, es preciso cuidar aspectos técnicos fundamentales en la eficiencia del examen en línea para el usuario, relacionados con sus particularidades, como su edad, experiencia, etapa de desarrollo cognitivo y psicomotriz (Karim y Shukur, 2016). Finalmente, se deben prever aspectos básicos de seguridad para las evaluaciones, por lo que aún hay trabajo por hacer en la mejora de los métodos de autenticación de usuario en un entorno en línea (Karim y Shukur, 2015).

Por otra parte, es importante señalar que la innovación con aprendizaje móvil es el proceso de enseñanza-aprendizaje formal o informal, que ocurre cuando el aprendiz no se encuentra en una ubicación fija, mediante el uso de dispositivos móviles como las PC, lectores MP3, tabletas, teléfonos inteligentes (smartphones) y móviles (UNESCO, 2016), así como recursos didácticos digitales innovadores como Socrative, Weebly, Quizlet, Jotform, Wolframalpha, Winksite, Classmarker, screencast-o-matic y Exploriments, que ofrecen formas originales para la administración de procesos, materiales, evaluaciones para distribuir información e interacción entre los docentes y alumnos, para el logro de los objetivos didácticos preestablecidos. Esto podría incentivar un cambio favorable en el proceso educativo formal e informal, como es el caso de los cursos abiertos masivos en línea, mediados por el uso de dispositivos móviles (MobiMOOC), nuevo estilo de adquisición de conocimientos que requiere el desarrollo de habilidades como la autoorganización y componentes como la interconexión y la apertura (De Waard, Abajian, Gallagher, Hogue, Keskin, Koutropoulos y Rodríguez, 2011).

Es así como la tecnología móvil se ha convertido en una herramienta de productividad, que incentiva la participación, creación y colaboración, por lo que es importante replantear los actuales paradigmas educativos, que ya no se encuentran limitados por barreras físicas, ni temporales (Shippee y Keengwe, 2014). El aprendizaje móvil crea un espacio para fomentar una cultura de participación, donde los estudiantes y docentes, por igual, pueden participar en esfuerzos conjuntos, con el propósito de obtener mejores resultados. Dos de estos factores 
críticos de éxito, de acuerdo con Cochrane (2014), son: la necesidad de nuevos enfoques para el soporte tanto técnico como pedagógico y el requerimiento de apoyo sostenido a las prácticas de trabajo colaborativo.

En este sentido, los procesos formativos con tecnología móvil pueden clasificarse en torno a las teorías clásicas de enseñanza: conductivista, constructivista, aprendizaje situado, aprendizaje colaborativo, informal y apoyarse en el uso de dispositivos móviles (Sandoval, García y Ramírez, 2012). Al verse implicadas distintas tareas en una sola actividad en el m-learning, se debe tomar en cuenta, para su evaluación, tanto la teoría de la disciplina como la forma en que los educandos se relacionan con la tecnología. De esta forma, la evaluación se clasifica en distintos niveles, dependiendo del grado de interacción y el impacto de la nueva tecnología; algunos instrumentos para evaluarla comprenden paquetes de software, registros de bases de datos, conversaciones de chat, exámenes y pruebas en línea, entre otros (Ozdamly y Cavus, 2011).

\section{Estrategia metodológica o didáctica}

Para abordar el problema de investigación, se formuló una interrogante que derivó del marco conceptual: ¿Cómo desarrolla las estrategias de aprendizaje del m-learning la alfabetización digital, en un curso de educación a distancia? En concordancia con la naturaleza de esta pregunta, se eligió como método de investigación el paradigma cualitativo, que, de acuerdo con Creswell (2012), es el más apropiado para, posteriormente, analizar e interpretar su significado, a partir de reflexiones y de la propia indagación. Además, se eligió el enfoque de estudio de casos, ya que permite dar respuesta a las interrogantes de cómo y porqué, al igual que permite comprender casos importantes a través de situaciones personales contextualizadas y la secuencia de acontecimientos (Yin, 2009). Para ello, se siguieron cinco pasos: se identificaron las fuentes de información y los sitios; se solicitó autorización del director; se determinaron los datos por recoger; se elaboraron los instrumentos, y, finalmente, se realizó una prueba piloto para verificar el funcionamiento de estos, para luego colectar y analizar la información obtenida (Stake, 1999).

El trabajo de campo se realizó en un instituto de estudios superiores localizado en Miami (Estados Unidos), que ofrece programas flexibles mediante educación en línea, donde el $80 \%$ de los alumnos 
son mexicanos. Para poder medir los resultados de la implementación de una estrategia didáctica y recursos móviles, se actualizó el programa de estudios, se diseñó un nuevo e-book y se introdujeron actividades interactivas de aprendizaje y repaso en la plataforma Moodle. Estas últimas se montaron en una storyline con la herramienta de tecnología educativa llamada SCORM (Sharable Content Object Reference Model, por sus siglas en inglés). Las categorías y los indicadores por indagar fueron: 1) alfabetización digital en educación a distancia, con los indicadores habilidades de la alfabetización digital, tipos de alfabetización digital y evaluación de la alfabetización digital; 2) innovación con aprendizaje móvil, con los indicadores innovación con aprendizaje móvil, aprendizaje vinculado a la tecnología móvil y habilidades que se desarrollan con el m-learning y 3) Aplicación didáctica de recursos de aprendizaje móvil, con los indicadores motivación del aprendizaje, rendimiento académico tras la introducción de recursos digitales y evaluación de integración de un recurso digital al diseño instruccional de un curso en línea.

La elección de los sujetos de análisis se caracterizó por basarse en un propósito definido y porque contiene elementos de pragmatismo como los límites de tiempo y de recursos (Descombe, 2003). Por lo tanto, en el estudio, fueron elegidos mediante el método de conveniencia, para seleccionar por el tiempo y localización a distancia de los informantes claves y demás fuentes de información, conformados por una muestra de ochenta y dos alumnos, un profesor, un diseñador instruccional y dos directivos localizados en diferentes países: México, Estados Unidos y Argentina.

Para extraer la información, se diseñaron los siguientes instrumentos: una entrevista en línea a directivos y un cuestionario autoadministrado a los alumnos. Se analizaron, además, por una parte, los datos registrados por el docente en la bitácora, donde fue documentado el proceso de implementación de la innovación, y, por la otra, los documentos significativos consistentes en los trabajos de los alumnos depositados en la plataforma.

Los resultados se capturaron textualmente, se codificaron por tema para poder tratar los datos a continuación, lo cual requirió organizarlos, analizarlos, desglosarlos, sintetizarlos e identificarlos por temas o categorías de respuestas. Para ello, se siguió la metodología de Stake (1999), lo que implicó secuenciar la acción, categorizar las propiedades 
y hacer recuentos para sumarlos de forma intuitiva, ya que, de acuerdo con el autor, el análisis se puede realizar mediante la interpretación directa de ejemplos individuales, o bien por la suma de ejemplos hasta que se pueda decir algo sobre ellos como conjunto o clase. Finalmente, se pudo analizar la información mediante interpretación directa, puesto que, como señala Denzin (1970), no hay datos puros en la indagación cuando se trata del comportamiento humano, lo cual es una característica propia de la investigación social.

Ahora bien, para poder desarrollar ideas contundentes que sirvieran para formular las conclusiones del estudio, se triangularon los datos obtenidos con la literatura previamente revisada en el marco teórico y los estudios analizados. Con esa finalidad, se aplicó la metodología señalada por Creswell (2012), se elaboró un cuadro de triple entrada que permitió cruzar la información de forma ordenada y analizarla a mayor profundidad. Cabe señalar que, en este sentido, la triangulación de datos refiere al proceso sistemático de clasificación para encontrar temas comunes, que, acorde con Creswell y Miller (2000), es un proceso fundamental para asegurar la validez y corroborar la evidencia recopilada. Por lo anterior, también se cumplieron dos estrategias propuestas por Stake (1999) que consisten en localizar información de las respuestas de cada informante y su coincidencia con los criterios de evaluación previamente establecidos, para después interpretar cada caso en forma individual y entonces buscar conexiones entre ellos.

Posteriormente, se documentó de modo explícito el proceso de investigación, para dejar registros de los procedimientos de recogida de datos y de los tres niveles de información: los datos inmediatos, el contexto, el programa tratado en la presente investigación, para la consiguiente comprensión del fenómeno en un entorno social más extenso. Todo ello, para poder cubrir lo expresado por Mathison (1988), quien señala que, si estos niveles de información son lo suficientemente sólidos, la verosimilitud y lógica de las explicaciones queda abierta a discusión de forma pública, lo que es también un criterio mínimo por cumplir en la indagación de las ciencias sociales.

Por último, para dar mayor confiabilidad a las conclusiones obtenidas y validar los instrumentos, los materiales, el proceso y los datos obtenidos, se siguieron cuatro cuestiones importantes para Lincoln y Guba (1985): que el proceso sea claro y cuidadosamente revisado desde distintas perspectivas para que sea creíble; permitir que el juicio sea 
replicable, para lo que se requiere proporcionar una base de datos; que el estudio pueda ser replicado en contextos distintos, y que sea confirmable. Lo que en este estudio dio sentido a la evidencia validó los datos y aseguró la confiabilidad de las conclusiones obtenidas, por lo que, a continuación, se presentan los resultados logrados y la discusión.

\section{Resultados y discusión}

Una vez aplicados los instrumentos, se analizaron e interpretaron los datos obtenidos, para lo cual se realizó una suma categórica como lo indica Stake (1999): los resultados contrastados con la información teórica, en concordancia con las categorías mencionadas. De esta forma, se aseguraron tanto la confiabilidad como la validez de los resultados, que se presentan seguidamente, ordenados por categoría de información.

\section{Alfabetización digital en educación a distancia}

Se identificó, por medio de la bitácora de observación, que el docente en la alfabetización digital debe de guiar y administrar el proceso de aprendizaje, de manera sucinta, y el alumno, desarrollar un estilo de aprendizaje autorregulado y disciplinado. Además, mediante el cuestionario a los alumnos, se encontró que la computadora portátil sigue siendo el dispositivo más utilizado para realizar actividades académicas, seguido por las tabletas; las herramientas informáticas a las que recurren con mayor frecuencia los alumnos son fuentes no académicas como los buscadores y blogs; los materiales didácticos de mayor utilidad fueron los videos y los textos teóricos; y las principales dificultades para aprender en línea son, por un lado, la ausencia de un profesor a quien manifestar sus dudas y, por el otro, la falta de hábito para estudiar por cuenta propia, principalmente (ver tabla 1). 


\section{Tabla 1.}

\section{Resultados del cuestionario electrónico respecto a la alfabetización digital}

\begin{tabular}{|c|c|}
\hline Consulta & Resultados \\
\hline $\begin{array}{l}\text { Dispositivos utilizados para acceder } \\
\text { al curso en línea }\end{array}$ & $\begin{array}{l}\text { Laptop }(97.7 \%) \text {; tableta }(18.5 \%) \text {; } \\
\text { teléfono inteligente }(6.6 \%)\end{array}$ \\
\hline $\begin{array}{l}\text { Herramientas de informática } \\
\text { integradas al proceso de enseñanza- } \\
\text { aprendizaje }\end{array}$ & $\begin{array}{l}\text { Buscadores }(74.7 \%) \text {; blogs }(32.9 \\
\%) \text {; aplicaciones para el celular }(16.6 \\
\%) \text {; software especializado }(8.9 \%)\end{array}$ \\
\hline $\begin{array}{l}\text { Materiales didácticos de mayor } \\
\text { utilidad para el proceso de } \\
\text { aprendizaje }\end{array}$ & $\begin{array}{l}\text { Videos }(71.6 \%) \text {; material teórico } \\
(71.6 \%) \text {; actividades de repaso }(36.5 \\
\%) \text {; apoyo del tutor }(27 \%) \text {; recetario } \\
\text { electrónico }(11 \%)\end{array}$ \\
\hline $\begin{array}{l}\text { Dificultades para aprender en el } \\
\text { curso en línea }\end{array}$ & $\begin{array}{l}\text { Ausencia del profesor }(55.4 \%) \text {; falta } \\
\text { de hábito para estudiar por cuenta } \\
\text { propia }(37.5 \%) \text {; la calidad de la } \\
\text { conexión a Internet }(21.4 \%) \text {; no tener } \\
\text { servicio de Internet en casa }(7.14 \%)\end{array}$ \\
\hline
\end{tabular}

Fuente: Elaboración propia, con base en las respuestas del cuestionario autoadministrado aplicado a los alumnos.

Asimismo, se encontró que el SCORM y las nuevas actividades didácticas lograron el uso de habilidades cognitivas y digitales de orden superior. También se pudo percibir que los participantes cada vez están más habituados a la educación virtual, como lo señaló uno de los estudiantes: "He tenido buenas experiencias en algunos cursos, un ejemplo de ello son los cursos en línea y gratuitos por tres días de $e d u K$ de los cuales he tomado varios" (Estudiante n. 1, 2017).

\section{Innovación con aprendizaje móvil}

Los alumnos identifican el storyboard como un recurso de apoyo al aprendizaje, como lo expresó uno de los alumnos: "Las diapositivas con la voz del profesor son de gran ayuda, te explica con detalle la presentación y es más gráfico, lo que permite hacer conexiones con los diferentes conocimientos, analizar el contenido y reforzar el aprendizaje" (Estudiante n. 2, 2017). Asimismo, el principal cambio que perciben los alumnos en su rol, mediante el aprendizaje móvil, es que deben ser 
autosuficientes en la forma de buscar información y aprender. Por otra parte, los beneficios reportados son: continuar sus estudios y consultar información en cualquier lugar (ver tabla 2).

Tabla 2.

Concentrado de los modelos en innovación con aprendizaje móvil

\begin{tabular}{|l|l|c|}
\hline \multicolumn{1}{|c|}{ Categoría B } & \multicolumn{1}{|c|}{ Modelos o patrones encontrados } & \% \\
\hline \multirow{2}{*}{$\begin{array}{l}\text { Rol de los alumnos } \\
\text { en el aprendizaje } \\
\text { móvil }\end{array}$} & $\begin{array}{l}\text { Ser autosuficiente en la forma de buscar } \\
\text { información y aprender }\end{array}$ & 60 \\
\cline { 2 - 3 } & $\begin{array}{l}\text { Rol activo de participación en el aprendizaje } \\
\text { con la guía del profesor }\end{array}$ & 30 \\
\cline { 2 - 3 } & Ser autodidacta & 30 \\
\hline \multirow{2}{*}{$\begin{array}{l}\text { Beneficios en el } \\
\text { aprendizaje con el } \\
\text { uso de la tecnología } \\
\text { móvil }\end{array}$} & Poder trabajar y estudiar al mismo tiempo & 80 \\
\cline { 2 - 3 } & $\begin{array}{l}\text { El acceso a Internet es más práctico por medio } \\
\text { de un teléfono inteligente }\end{array}$ & 10 \\
\hline
\end{tabular}

Fuente: Elaboración propia, con base en las respuestas del cuestionario autoadministrado aplicado a los alumnos.

Por añadidura, la transmisión de contenidos digitales para adquirir habilidades prácticas y la obtención de información actualizada son beneficios que ha aportado la nueva tecnología en los cursos a distancia y móviles; en especial, una estudiante manifestó: "Se tiene acceso a diferentes páginas con información actual, moderna, nuevas tendencias provenientes de todo el mundo" (Estudiante n. 3, 2017).

\section{Aplicación didáctica de recursos de aprendizaje móvil}

En la entrevista a los directivos, se encontró que el SCORM permite comprobar la adquisición del conocimiento y consolidarlo a largo plazo. En la integración del recurso digital al diseño instruccional del curso en línea, se halló que: 1) la experiencia con los recursos móviles aún presenta áreas técnicas de mejora; 2) con el afán de introducir los recursos digitales, se debe hacer un diseño web adaptable o Responsive Web Design, para que se logre una visualización adecuada en cualquier dispositivo en el que se acceda; 3 ) el uso de la storyline implica la adaptación de dinámicas compatibles con el tipo de animación requerido. 
No obstante, el recurso digital permite facilitar al alumno la experiencia de aprendizaje y, en consecuencia, los directivos esperan, con su implementación, aumentar los índices de graduación y reducir las tasas de abandono. Por otra parte, la percepción de la experiencia con el uso de los recursos móviles ha sido buena en el $70 \%$ de los alumnos.

Tabla 3.

Concentrado de modelos de aplicación didáctica de recursos móviles

\begin{tabular}{|c|c|c|}
\hline Categoría C & Modelos o patrones encontrados & $\%$ \\
\hline \multirow{3}{*}{$\begin{array}{l}\text { Percepción de la } \\
\text { experiencia con el uso de } \\
\text { los recursos móviles }\end{array}$} & Buena & 70 \\
\hline & Regular & 30 \\
\hline & Mala & 0 \\
\hline \multirow{6}{*}{$\begin{array}{l}\text { Estrategias de aprendizaje } \\
\text { adoptadas gracias a } \\
\text { los recursos didácticos } \\
\text { utilizados en el curso en } \\
\text { línea }\end{array}$} & $\begin{array}{l}\text { Realizar búsquedas especializadas en } \\
\text { Internet }\end{array}$ & 60 \\
\hline & $\begin{array}{l}\text { Habilidad de analizar y validar } \\
\text { conceptos }\end{array}$ & 50 \\
\hline & $\begin{array}{l}\text { Capacidad de trabajar } \\
\text { colaborativamente en la red }\end{array}$ & 30 \\
\hline & $\begin{array}{l}\text { Habilidad para diseñar y analizar } \\
\text { documentos electrónicos }\end{array}$ & 30 \\
\hline & $\begin{array}{l}\text { Habilidad para suscribirse a páginas } \\
\text { especializadas }\end{array}$ & 20 \\
\hline & $\begin{array}{l}\text { Capacidad de subir archivos a un } \\
\text { servidor }\end{array}$ & 10 \\
\hline
\end{tabular}

Fuente: Elaboración propia, con base en las respuestas del cuestionario autoadministrado aplicado a los alumnos.

Por último, las estrategias de aprendizaje adoptadas gracias a los recursos didácticos utilizados fueron, principalmente, realizar búsquedas especializadas en la web, con un $60 \%$ de incidencia, seguida por la habilidad para análizar de los conceptos encontrados, en un $50 \%$ de los casos.

\section{Conclusiones}

Tomando en consideración lo expresado en líneas anteriores, la respuesta concreta a la pregunta de investigación ¿Cómo desarrolla las estrategias de aprendizaje del m-learning la alfabetización digital, en un curso de educación a distancia? se puede agrupar en tres puntos principales: 
1. La alfabetización digital se desarrolla por medio de la implementación de estrategias de aprendizaje móvil que incrementan el grado de dominio sobre las habilidades tecnológicas y éticas, en la búsqueda y el uso de la información, así como en el manejo de dispositivos móviles, como la storyline de la tecnología SCORM, con el objetivo explícito de enriquecer el proceso de enseñanza-aprendizaje y desarrollar actividades académicas.

2. El uso de los dispositivos móviles con fines didácticos ayuda a desarrollar habilidades digitales importantes para la alfabetización digital, como la transformación de datos en conocimiento, con base en navegaciones no lineales de hipertexto, la lectura en monitores gráficos y la realización de búsquedas avanzadas de material especializado con fines académicos, más la capacidad de utilizarlo correctamente.

3. Por último, se incorpora en los cursos modelos evaluativos con indicadores que midan específicamente destrezas propias de la inclusión digital, como el manejo de redes digitales, la lectura digital, el uso de las TIC, la forma en que se accede a la información y la ética en el manejo de esta.

Ahora bien, la información descrita en líneas anteriores confirma que el m-learning es un estilo de aprendizaje que crea condiciones propicias para que los estudiantes generen procesos de reflexión, análisis y apropiación tecnológica, además del desarrollo de competencias transversales como la curiosidad, disciplina, autorregulación, autosuficiencia en el aprendizaje. La importancia de conocer de forma específica las competencias desarrolladas permite, por un lado, revelar los beneficios del uso de las TIC en la educación, para mostrar a la comunidad docente que pueden ser aliados de su labor educativa, y, por otro, permite tomar mejores decisiones a diseñadores instruccionales, docentes y directivos de Instituciones de Educación, para diseñar e implementar recursos didácticos móviles en cursos presenciales o a distancia.

También se pudieron observar áreas de mejora técnica en los dispositivos móviles, como la incompatibilidad de tecnologías, el tamaño de la pantalla, la velocidad de reproducción de los materiales, la falta de capacidad de almacenamiento de información y la adaptabilidad de los gráficos al formato de los dispositivos. En el mismo sentido, aún hay limitantes tanto por parte de los docentes como de los estudiantes, 
en el hábito de usar, con fines académicos, los dispositivos móviles, la brecha digital y la capacidad de lectura de comprensión en monitores pequeños. Sin embargo, se espera que estas limitantes cambien en el futuro cercano, pues las habilidades digitales se desarrollan y evolucionan rápidamente en las nuevas generaciones. Por ello, se recomienda a los responsables de los programas que utilicen recursos didácticos de m-learning para acompañar sus materiales, con tutoriales que permitan al usuario familiarizarse más rápidamente en el manejo de los dispositivos móviles en el ámbito formativo y seguir haciendo investigación educativa, la cual permita conocer los avances en el grado de dominio de los recursos digitales.

Por otra parte, se recomienda a los docentes y diseñadores instruccionales promover, de forma intencional, el uso de competencias digitales y transversales, que permitan asegurar la calidad educativa e instar la adquisición de conocimiento a largo plazo. Para lo anterior, es necesario estimular habilidades como recordar, comprender, enlazar conceptos y hacer lo mismo con documentos electrónicos; así, posteriormente, se estará en condiciones de desarrollar habilidades de orden superior que motiven la aplicación del conocimiento, como evaluar, analizar y crear tanto información como materiales digitales. Finalmente, se sugiere realizar más investigaciones respecto a estrategias que permitan hacer más cercana y humanizada la experiencia de aprendizaje a través del m-learning, sobre todo la resolución de dudas en tiempo real; asimismo, indagar respecto a qué dispositivos móviles son los idóneos desde el punto de vista de los usuarios y por qué, para poder, con esta información, potencializar su aprovechamiento didáctico.

\section{Referencias}

Álvarez, G. y Álvarez, G. (2012). Análisis de ambientes virtuales de aprendizaje desde una propuesta semiótico integral. Revista electrónica de investigación educativa, 14(2), 73-88. Recuperado de http://www.scielo.org.mx/scielo. php?pid=S1607-40412012000200006\&script=sci_arttext

Barroso, J., Cabero, J. y Vázquez, A. (2012). La formación desde la perspectiva de los entornos personales de aprendizaje (PLE). Revista Apertura, 4(1), 6-19. Recuperado de http://www.udgvirtual. udg.mx/apertura/index.php/apertura/article/view/209 
Belshaw, D. (2012). What is digital literacy'? A Pragmatic investigation (Disertación doctoral). Durham, Reino Unido: Faculty of Social. Sciences and Health, Durham University, Recuperado de $\mathrm{http}: / /$ neverendingthesis.com/doug-belshaw-edd-thesis-final.pdf

Ciampa, K. (2013). Learning in a mobile age: an investigation of student motivation. Journal of Computer Assisted Learning, 30(1), 82-96. Doi: $10.1111 /$ jcal.12036

Cobo, C. y Moravec, J. (2011). Introducción al aprendizaje invisible: la (r)evolución fuera del aula. Reencuentro Análisis de Problemas Universitarios, 62, 66-81. Recuperado de http://www.redalyc. org $/ \mathrm{html} / 340 / 34021066008 /$

Cochrane, T. D. (2014). Critical success factors for transforming pedagogy with mobile Web 2.0. British Journal of Educational Technology, 45(1), 65-82. Doi: 10.1111/j.1467-8535.2012.01384.x

Conde, M. Á., García-Peñalvo, F. J., Alier, M. y Piguillem, J. (2013). The implementation, deployment and evaluation of a mobile personal learning environment. Journal of Universal Computer Science, 19(7), 854-872. Recuperado de http://jucs.org/jucs_19_7/the_implementation_deployment and/jucs_19_07_0854_0872_conde.pdf

Creswell, J. W. (2002). Educational research: Planning, conducting, and evaluating quantitative and qualitative research (pp. 146-166). Upper Saddle River, NJ: Prentice Hall. Recuperado de http://www. onlinecef.net/file.php/1/CEF_Resources/Research\%20\%20Method/_Educational_Research_Planning_Conducting_and_Evaluating_Quantitative_and_Qualitative_Research_4th_Edition_.pdf

Creswell, J. y Miller, D. (2000). Determining validity in qualitative inquiry. Theory into practice, 39(3), 124-130. Doi: 10.1207/ s15430421tip3903_2

Cuevas, A. y García, M. (2010). Ideias, un modelo de evaluación para inclusión digital y alfabetización informacional orientado a salud. El profesional de la información, 19(3), 240-245. Doi: 10.3145/ epi.2010.may.03

De Waard, I., Abajian, S., Gallagher, M. S., Hogue, R., Keskin, N., Koutropoulos, A. y Rodríguez, O. C. (2011). Using mLearning and MOOCs to understand chaos, emergence, and complexity in education. International Review of Research in Open and Distance Learning, 12(7), 94-115. Recuperado de http://www.irrodl. org/index.php/irrodl/article/view/1046 
Denscombe, M. (2003). The good research guide. Philadelphia: McGraw-Hill Education. Recuperado de http://iwansuharyanto. files.wordpress.com/2013/04/martyn_denscombe_the_good_research_guidebookfi-org.pdf

Denzin, N. (1970). The Research Act: A Theoretical Introduction to Sociological Methods. Chicago: Aldine. Recuperado de http:// www.albany.edu/ scifraud/data/sci_fraud_1437.html

Didou, S. (2014). La UNESCO y la educación superior, 2014-2017: aportes de la Reunión de Cátedras UNESCO sobre la educación superior, las TIC en la educación y los profesores. París, Francia: UNESCO. Recuperado de http://www.unesco.org/new/fileadmin/MULTIMEDIA/HQ/ED/pdf/UNESCO-summary-report-chairs-2014-1.pdf

Figueredo, O. y Jiménez J. (2015). Estrategias de aprendizaje móvil, una propuesta teórica para su diseño. Memorias del Encuentro Virtual Educa VE2003. Guadalajara, México. Recuperado de http://reposital.cuaed.unam.mx:8080/jspui/bitstream/123456789/3891/1/VE13.394.pdf

García-Holgado, A. y García-Peñalvo, F. J. (2016). Architectural pattern to improve the definition and implementation of eLearning ecosystems. Science of Computer Programming, 129, 20-34. Doi: 10.1016/j.scico.2016.03.010

González-Bañales, D. y Monárrez-Armendáriz, C. (2014). Incorporación de redes sociales y aplicación de principios de diseño adaptativo para la plataforma moodle. ITECKNE: Innovación e Investigación en Ingeniería, 11(1), 50-61. Recuperado de http:// www.scielo.org.co/pdf/itec/v11n1/v11n1a06.pdf

Humanante-Ramos, P., García-Peñalvo, F. y Conde-González, M. (2016). PLEs en contextos móviles: Nuevas formas para personalizar el aprendizaje. Versión Abierta Español-Portugués, VAEP-RITA, 4, 33-39. Recuperado de https://www.researchgate. net/profile/Francisco_Garcia-Penalvo/publication/304024281 PLEs_en_contextos_moviles_Nuevas_formas_para_personalizar_el_aprendizaje/links/5763d0ed08aeb4b997fc8482.pdf

Jiménez-Hernández, C., Castellanos-Domínguez, O. y Villa-Enciso, E. (2011). La Gestión de Tecnologías Emergentes en el Ámbito Universitario. Tecno Lógicas, 26, 145163. Recuperado de http://www.scielo.org.co/scielo. php?pid=S0123-77992011000100009\&script=sci_arttext 
Karim, N. A. y Shukur, Z. (2015). Review of user authentication methods in online examination. Asian Journal of Information Technology, 14(5), 166-175. Recuperado de http://docsdrive.com/ pdfs/medwelljournals/ajit/2015/166-175.pdf

Karim, N. A. y Shukur, Z. (2016). Proposed features of an online examination interface design and its optimal values. Computers in Human Behavior, 64, 414-422. Doi: 10.1016/j.chb.2016.07.013

Lincoln, Y. y Guba, E. (1985). Naturalistic inquiry. Thousand Oaks, California: Sage.

Mathison, S. (1988). Why triangulate? Educational Researcher, 17(2), 13-17. Doi: 10.3102/0013189X017002013

Newland, B. y Handley, F. (2016). Developing the digital literacies of academic staff: an institutional approach. Research in Learning Technology,24(1), 31501. Doi: 10.1080/21567069.2017.1270579

Organista-Sandoval, J., Serrano-Santoyo, A., McAnally-Salas, L. y Lavigne, G. (2013). Apropiación y usos educativos del celular por estudiantes y docentes universitarios. Revista electrónica de investigación educativa, 15(3), 139-156. Recuperado de http:// www.scielo.org.mx/scielo.php?pid=S1607-4041201300030001 $0 \&$ script $=$ sci_arttext

Pathak, B. K. (2016). Emerging online educational models and the transformation of traditional universities. Document Electronic Markets, 26(4), 315-32. Doi: 10.1007/s12525-016-0223-4

Ramos, A. I., Herrera, J. A. y Ramírez, M. S. (2010). Desarrollo de habilidades cognitivas con aprendizaje móvil: un estudio de casos. Comunicar: Revista Científica Iberoamericana de Comunicación y Educación, 17(34), 201-209. Doi: 10.3916/C34-2010-03-20

Sandoval, E. A., García, R. y Ramírez, M. S. (2012). Competencias tecnológicas y de contenido necesarias para capacitar en la producción de recursos de aprendizaje móvil. Edutec-e: Revista Electrónica de Tecnología Educativa, 39, 1-16. Doi: 10.21556/ edutec.2012.39.379

Shippee, M. y Keengwe, J. (2014). mLearning: Anytime, anywhere learning transcending the boundaries of the educational box. Education and Information Technologies, 19(1), 103-113. Doi: 10.1007/s10639-012-9211-2

Stake, R. (1999). Investigación con estudio de casos. Madrid, España: Morata. 
UNESCO (2011). El Aprendizaje Móvil. First Mobile Learning Week Report. Recuperado de http://www.unesco.org/new/fileadmin/ MULTIMEDIA/HQ/ED/ICT/pdf/UNESCO\%20MLW\%20report $\% 20$ final $\% 2019$ jan.pdf

UNESCO (2016). Las TIC en la Educación, el aprendizaje móvil. Recuperado de http://www.unesco.org/new/es/unesco/themes/icts/m4ed/

Waheed, M. (2016). What role does knowledge quality play in online students' satisfaction, learning and loyalty? An empirical investigation in an eLearning context: Learning and loyalty: role of KQ. Journal of Computer Assisted Learning, 32(6), 561-575. Doi: 10.1111/jcal.12153

Yin, R. (2009). Case Study Research (4. ${ }^{\mathrm{a}}$ ed.). California, Estados Unidos: Sage.

Zatarain, R. y Barrón, M. L. (2011). Herramienta de autor para la identificación de estilos de aprendizaje utilizando mapas auto-organizados en dispositivos móviles. Revista electrónica de investigación educativa, 13(1), 43-55. Recuperado de http://www.scielo. org.mx/scielo.php?pid=S1607-40412011000100003\&script=sci_arttext\&tlng=pt

Zezulkova, M. (2017). Digital Competence and Capability Frameworks in the Context of Learning, Self-Development and HE Pedagogy. En E-Learning, E-Education, and Online Training (pp. 46-53). Springer, Cham. Doi: 10.1007/978-3-319-49625-2_6 